\title{
Analysis based on the diffusion model for saturation silica gel with water vapor at conservation units steam circuit TPP
}

\author{
Sergey Goldaev ${ }^{1}$ and Alisher Khushvaktov ${ }^{2, a}$ \\ ${ }^{1}$ Dr. Sci. (Phys. -Math.) National Research Tomsk Polytechnic University, 634050 Tomsk, Russia \\ ${ }^{2}$ Postgraduate, Tajik Technical University, 734042 Dushanbe, Tajikistan
}

\begin{abstract}
A quantitative analysis of the diffusion model dehumidifying air in the steam circuit of TPP, with a layer of silica gel. Showed that such an approximation, supplemented the experimental value of the coefficient of free diffusion identified by the developed method gives reliable values for the concentration of water vapor absorption over time.
\end{abstract}

Operating experience TPP shows that when carrying out low-quality preservation of their units, the condensation of moisture contained in the air and gases during shutdown with reduced environmental pressure to atmospheric, and hit the interior of the oxygen in the air causes the parking atmospheric corrosion (PAC) of carbon steel [1].

Effective solution of the problem of multilateral defense units steam circuit (USC) TPP with downtime from PAC will keep their endurance, reduce the material loss due to corrosion of metals, reduce environmental pollution [2].

In [3] proposed to use for the preservation of USC TPP silica gel, which is widely used in the process of cleaning the flue gases of carbon dioxide [4], in adsorbers for air separation plants [5], and others. It is produced on an industrial scale and has a lower rate [6].

When USC TPP conservation, maintaining a predetermined humidity in closed volumes, sufficiently widespread drainage in static conditions. The amount of the adsorbent loaded in this case is mainly determined equilibrium adsorption capacity. Process silica moisture saturation under static conditions characterized by a low rate. The duration of complete saturation at room temperature, small-pore silica is 40 hours average pore -50 hours and large pores -75 hours [4, 7]. A fixed relative humidity of the gas moisture content $\left(g_{c}\right)$ of silica gel is not dependent on the temperature at which the absorption process [8].

In the theory of adsorption are used various nonstationary setting [5, 9]. When the process is considered in a time interval in which the concentration $(\mathrm{C})$ at the opposite end from the open surface of the particles or at its center with a symmetric mass transfer is not yet time to change significantly, such that is applicable mathematical formulation of [9].

$$
\frac{\partial C}{\partial \tau}=D_{c} \frac{\partial^{2} C}{\partial x^{2}}
$$

\footnotetext{
${ }^{a}$ Corresponding author: alisher1001@mail.ru
}

This is an Open Access article distributed under the terms of the Creative Commons Attribution License 4.0, which permits unrestricted use, distribution, and reproduction in any medium, provided the original work is properly cited. 
Table 1. shows the average values of $D_{c}$ with an error of $5 \%$ for various silica gel granules.

\begin{tabular}{|c|c|c|}
\hline Solution & $d, \mathrm{~mm}$ & $D_{c} \cdot 10^{7}, \mathrm{~m}^{2} / \mathrm{c}$ \\
\hline \multirow{4}{*}{ Water } & 2,85 & 2,54 \\
\cline { 2 - 3 } & 3,65 & 2,92 \\
\cline { 2 - 3 } & 4,68 & 4,17 \\
\cline { 2 - 3 } & 5,80 & 5,01 \\
\hline
\end{tabular}

at

$$
\tau=0, C=0 ; x=0, C=C_{0} ; x \rightarrow \infty, C=0,
$$

here $D_{c}$ - free diffusion coefficient.

It is found that the assumption of semiboundness adsorbent sample thickness $L_{S}$ can be considered valid if the diffusion Fourier number $F o_{D}=D_{c} t / L_{s}^{2}<1,0[10]$.

The exact analytical solution of the problem (1)-(2) has the form [11]

$$
C(x, \tau)=C_{0}\left[1-\operatorname{erf}\left(\frac{x}{2 \sqrt{D_{c} \tau}}\right)\right]
$$

where $\operatorname{erf}(z)=(2 / \sqrt{\pi}) \int_{0}^{z} \exp \left(-y^{2}\right) d y$ - the probability integral function or Crump, which occurs in non-stationary theory of thermal conductivity [11], in the heat processing of metals [12].

For automated calculation of the distribution of the tabular method for determining the concentration of the probability integral laborious and more convenient to use interpolation expression, for example, is [12]:

$$
\operatorname{erf}(z)=\sqrt{1-\exp \left(-1,26 \cdot z^{2}\right)}
$$

Despite the fact that the formula contains only one constant, it is fairly accurate, error it does not exceed $1 \%$.

The described algorithm has been implemented in Turbo Pascal.

The values of the free diffusion defined by means of setting, developed at the department "Heat and heat engineering equipment" Tajik Technical University, which received a patent [13]. This device consists of a desiccator, electronic scales, Petri dishes, electronic stopwatch. Measurements were performed as follows: particulate material (silica-based nickel), depending on the size of the granules is divided into fractions (samples with different diameters): $2.85 \mathrm{~mm}, 3.65 \mathrm{~mm}, 4.68 \mathrm{~mm}, 5.8 \mathrm{~mm}$, weighed dry and placed in a desiccator containing a solution of the bottom, where the air is saturated by molecules of this solution. Over 10 hours, every 2 hours, the examined object is removed from the desiccator and weighed by the formula (4), the free diffusion coefficient is calculated:

$$
D_{c}=(l / \pi)^{2} \operatorname{tg}(\alpha) .
$$

Here $l$ - thickness of the sample; $\pi=3,14 ; \operatorname{tg}(\alpha)=\ln (1-\gamma) / t$ - is determined graphically from the slope of $\ln (1-\gamma)$ from $t_{\text {sek }}$ where $\gamma$ - swelling coefficient, calculated by the formula

$$
\gamma=M_{t} / M_{\infty} .
$$

Here $M_{t}$ - mass of the sample at time t, $M_{\infty}$ - maximum mass of the swollen sample. From the obtained experimental data, knowing $M_{\mathrm{t}}$, and $M_{\infty}$, by (5) is the coefficient of swelling [14].

In Table 1 shows the average values of $D_{c}$ with an error of $5 \%$ for various silica gel granules.

Figure 1 shows the concentration profiles of water vapor in the thickness of silica gel at a value of $D_{c}=2,54 \cdot 10^{-7} \mathrm{~m}^{2} / \mathrm{s}$ for different time points that satisfy the inequality $\mathrm{Fo}_{D}=D_{c} t / L_{s}^{2}<1,0$.

As can be seen, when the adsorption process is determined only by free diffusion, then the saturation of the silica gel layer is sufficiently slow. 


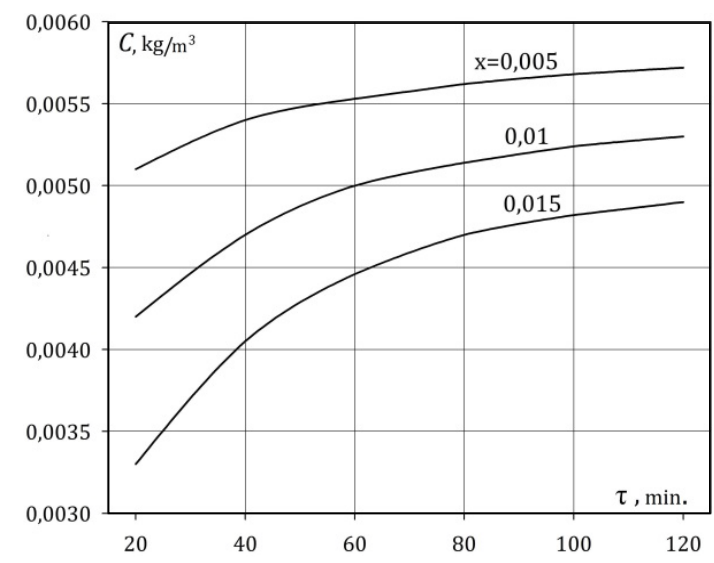

Figure 1. Concentration of water vapor from time to time in different sections of the silica gel sample.

These results agree qualitatively with the data of numerical calculations of the thickness of the silica gel, which is saturated with water vapor produced in the framework of other models [15].

Thus, the diffusion model of moisture absorption layer of silica gel, "closed" the experimental value of the coefficient of free diffusion determined by the developed method gives reliable values.

\section{References}

[1] G. P. Sutockij, Damage to the power equipment associated with water-chemical regime (S-Peterburg, "NPO CKTI" 1992)

[2] A. F. Bogachev, Thermal Engineering 7 (2001)

[3] S. V. Goldaev, A. A. Khushvaktov, Energy: Efficiency, reliability, safety: material works XIX Russian Sci. Tech. Conf. 2. 386-389 (2013)

[4] E. N. Serpionova, Industrial adsorption of gases and vapors (Moscow: "Hi school", 1969)

[5] V. P. Alekseev, G. E. Vainshtain, P. V. Gerasimov, Calculation and simulation of cryogenic devices (Energoatomizdat. Leningrad. 1987)

[6] Market Overview of silica gel in the CIS, http://www.infomineru/research/18/441 (15.10.2014)

[7] V. S. Morozov, E. V. Morozov, S. V. Vihrova, Technical gases 6 (2005)

[8] A. N. Ostrikov, Processes and equipment for food production (SPb.: GIORD. 2007)

[9] P. G. Romankov, V. F. Frolov, Mass transfer processes of chemical technology (L.: Khimiya, 1990)

[10] F. Kreyt, U. Blek, Fundamentals of heat transfer (M.: Mir, 1983)

[11] A. V. Lyikov, Heat and Mass Transfer: A Guide (M.: Energiya, 1972)

[12] A. N. Reznikov, A.N. Reznikov, Thermal processes in technological systems (M.: Mashinostroenie, 1990)

[13] A. A. Khushvaktov, M. M. Safarov, D. S. Juraev, Patent TJ 473. Tajikistan, MPK (2011.01) G 01 N5/02. A method of determining the coefficients of self-diffusion and mass transfer of dispersed materials $4 \mathrm{p}$.

[14] A. A. Khushvaktov, M. M. Safarov, Bulletin of the Tajik Technical University, 4 (2011)

[15] S.V. Goldaev, A. A. Khushvaktov, Of the universities. Energy problems 7-8 (2014) 